\title{
Microstructure Characterization of Microalloyed 5xxx Aluminum Alloys with Er and Zr using Analytical Transmission Electron Microscopy and Synchrotron X-ray Fluorescence Microscopy
}

Xiaolan $\mathrm{Wu}^{1,2}$, Si Chen ${ }^{2}$, Barry $\mathrm{Lai}^{2}$, Huang Hui ${ }^{1}$, Shengping Wen ${ }^{1}$, Kunyuan Gao ${ }^{1}$, Wei Wang ${ }^{1}$, Zuoren $\mathrm{Nie}^{1}$, and Nestor J. Zaluzec ${ }^{3}$

1. College of Materials Science and Engineering, Beijing University of Technology, Beijing, China.

2. X-ray Science Division, Argonne National Laboratory, Lemont, USA.

3. Photon Sciences Division, Argonne National Laboratory, Lemont, USA.

5xxx aluminum alloys with the major alloying element of $\mathrm{Mg}$, have been widely used as structural materials in all kinds of fields, due to their excellent strength, corrosion resistance, formability and weldability. As non-heat treatable alloys, their strength is derived mainly from the work hardening and the solid solution strengthening of $\mathrm{Mg}$ which has a substantial solid solubility in $\mathrm{Al}$ matrix. However, high $\mathrm{Mg}$ concentration in 5xxx alloys will cause processing challenges and increase of the susceptibility to stress corrosion cracking. It is well known that microalloying, namely addition of elements in trace amount, is an effective way to improve the comprehensive properties of aluminum alloys [1]. Recently, it has been proved that joint adding of rare earth element Er and transition element $\mathrm{Zr}$ into $\mathrm{Al}$ alloys can enhance the mechanical behaviors, thermal stability and corrosion resistance comprehensive significantly due to the formation of thermal stable $\mathrm{L1}_{2}$-ordered-structured $\mathrm{Al}_{3}(\mathrm{Er}, \mathrm{Zr})$ precipitates [2-4].

The work reported here focuses on a newly registered 5xxx alloy in China, named 5E61, with a nominal composition of Al-6Mg-0.9Mn-0.07Zr-0.2Er (wt.\%), which has a relatively high $\mathrm{Mg}$ concentration and microalloyed with Er and Zr. The microstructure and trace element distributions of this alloy have been characterized using an analytical electron microscope (AEM) equipped with $\mathrm{X}$-ray energy dispersive spectroscopy (XEDS) and synchrotron-based X-ray fluorescence microscopy (XFM) at the Advanced Photon Source (APS) of Argonne National Laboratory.

The experimental alloy was prepared by traditional casting in an iron mold and homogenized at $280^{\circ} \mathrm{C}$ for $10 \mathrm{~h}$ and then $460^{\circ} \mathrm{C}$ for $36 \mathrm{~h}$. After the homogenization, the ingot was hot-rolled to $8 \mathrm{~mm}$ thick $(\sim 70 \%$ rolling reduction) at $460^{\circ} \mathrm{C}$, followed by an intermediate annealing at $350^{\circ} \mathrm{C}$ for $2 \mathrm{~h}$. Subsequently, the sheet was cold-rolled down to $4 \mathrm{~mm}$ thick ( $\sim 50 \%$ rolling reduction) followed by annealing at $260^{\circ} \mathrm{C}$ for 4 $\mathrm{h}$. Thin foils for TEM analysis were prepared by electropolishing. AEM measurements were carried out on an FEI Talos 200kV S/TEM equipped with a SuperX quad spectrometer system. The X-ray induced X-ray fluorescence results were obtained at the Bionanoprobe and 2-ID-D microprobe at the APS.

Figure 1 presents the AEM XEDS color coded hyperspectral elemental imaging, as well as the annular dark field image from the corresponding area. Nanosized Er, Zr-enriched particles were observed the first time in these alloys (as arrowed), which are likely $\mathrm{L1}_{2}$-ordered-structured $\mathrm{Al}_{3}(\mathrm{Er}, \mathrm{Zr})$ precipitates as seen in Al-Er-Zr alloy [4]. Micron and submicron Er-enriched precipitates were also observed, and are presumably the phase produced during the casting due to the very limited solid solubility of Er in Al. Mg-enriched $\beta$ phases were also present as well as the rod-shaped Mn-enriched phases, which are likely $\mathrm{Al}_{6} \mathrm{Mn}$ primary phase, elongated along the rolling direction. Elemental distributions were also acquired using XFM from both the thin area measured on the AEM and a thicker region of the sample, with the later shown in Figure 2. Both Mn-enriched and Er-enriched phases, again are very likely the primary 
phases from the casting, and were observed ranging from a couple hundred nanometers to a few microns in size. While AEM offers unprecedented resolution to study micron to sub-nanoscale precipitates, the synchrotron-based XRM, without bremsstrahlung background, can in some cases provide a higher elemental sensitivity as well as survey larger and thicker regions of the sample, albeit at lower resolution. By combining the complimentary information obtained from the two techniques, we aim to understand the sample across multi scales. Further quantitative analysis and correlation are undergoing and will be reported separately.

\section{References}

[1] K Knipling, D Dunand, D Seidman, Zeitschrift Fur Metallkunde 97 (2006), p. 246.

[2] Z Nie, B Li, W Wang, Materials Science Forum 546-549 (2007), p. 623.

[3] S Wen, K Gao, Y Li, H Huang, et al., Scripta Materialia 65 (2011), p. 592.

[4] S Wen, K Gao, H Huang, W Wang, et al., Journal of Alloys and Compounds 574 (2013), p. 92.

[5] The authors acknowledge funding from the National Natural Science Foundation of China, Grant Number 51701006. This research used resources of the Advanced Photon Source, a U.S. Department of Energy (DOE) Office of Science User Facility and the Center for Nanoscale Materials, an Office of Science user facility, as well as support from Laboratory Directed Research and Development (LDRD) funding No. 2017-153-N0 at Argonne National Laboratory supported by the U.S. Department of Energy, Office of Science, Office of Basic Energy Sciences, under Contract No. DE-AC02-06CH11357.

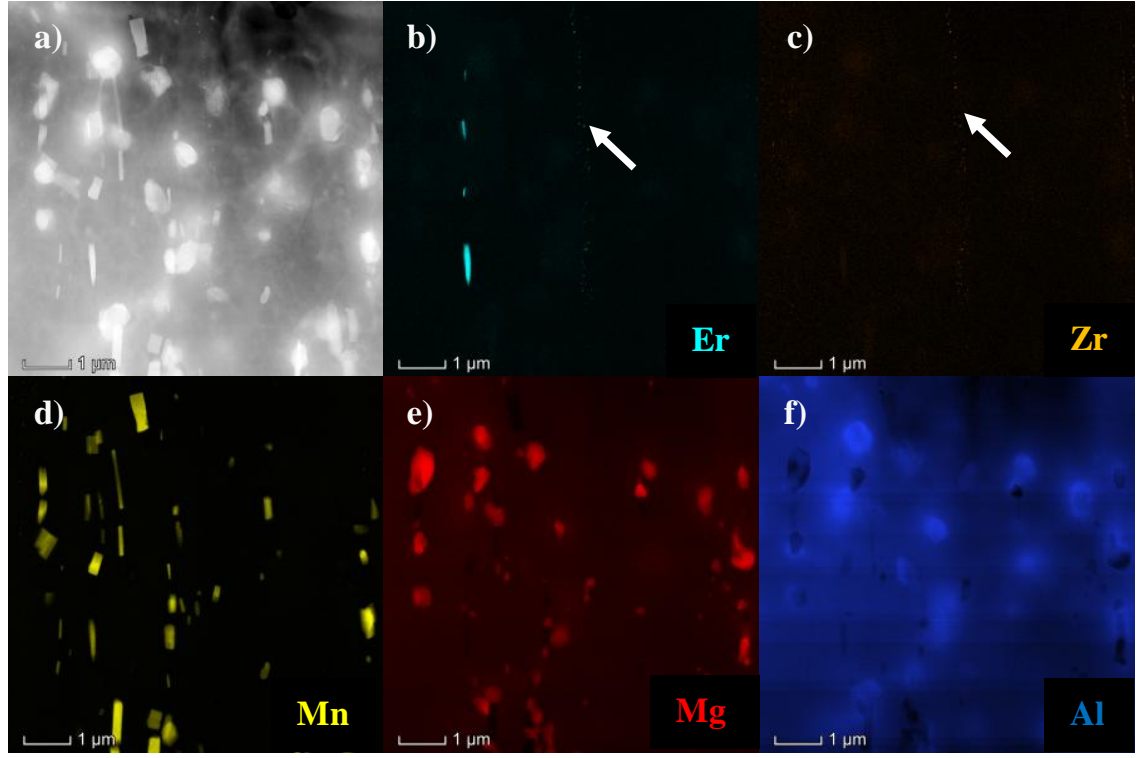

Figure 1. AEM results from the 5E61 alloy. a) HAADF image; hyperspectral images of b) Er; c) Zr; d) $\mathrm{Mn}$; e)Mg and f) Al. Conditions: Eo: $200 \mathrm{keV}, 4 \mathrm{~nm}$ pixel size, $3 \mathrm{~ms}$ dwell time per pixel

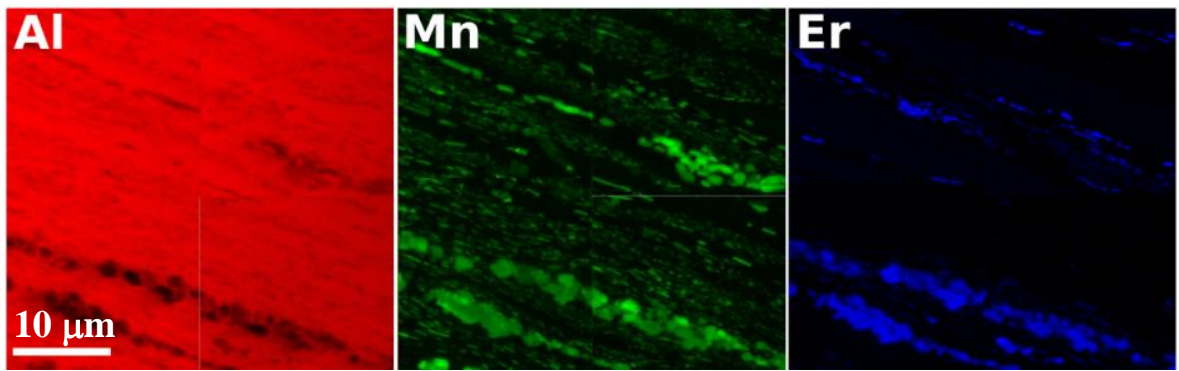

Figure 2. XRF results collected at the ANL Bionanoprobe with $10 \mathrm{keV}$ incident photons, which is not enough energy to be tuned to measure $\mathrm{Zr}, 80 \mathrm{~nm}$ scanning pixel size, $100 \mathrm{~ms}$ dwell time per pixel. 